\title{
APPROXIMATING HOMOTOPIES BY ISOTOPIES IN FRECHET MANIFOLDS
}

\author{
BY JAMES E. WEST
}

Communicated by Richard D. Anderson, April 18, 1969

Let $M$ be an $F$-manifold, that is, a separable, metric manifold modelled on an infinite-dimensional Fréchet space. The question was raised at a problem seminar this January (1969) at Cornell University whether homotopic embeddings of another $F$-manifold in $M$ are isotopic. In this note the affirmative answer is given and a stronger result established.

Given an open cover $\mathcal{U}$ of a space $X$, two maps $f$ and $g$ of a space $Y$ into $X$ are said to be $u$-close provided that for each $y$ in $Y$ there is an element of $u$ containing both $f(y)$ and $g(y)$. The two maps are said to be pseudo-isotopic provided there is a map $h: Y \times I \rightarrow X$ with

$$
h(y, 0)=f(y), \quad h(y, 1)=g(y)
$$

and which for each $t$ in $(0,1)$ is an embedding of $Y \times\{t\}$. The theorem is as follows:

THEOREM. Homotopic maps of a separable metric space into an F-manifold are pseudo-isotopic. If the domain is complete, the pseudoisotopy may be required to be through closed embeddings. Furthermore, given any open cover $u$ of the manifold and any homotopy $F$ between the maps, the pseudo-isotopy may be required to be u-close to $F$.

Proof. Let $X$ be the separable metric space, $M$ the $F$-manifold, and $f$ and $g$ the homotopic maps of $X$ into $M$. By a collection of results, all separable, infinite-dimensional Fréchet spaces are homeomorphic to the countably infinite product $s$ of open intervals $(-1,1)$. (For a discussion of these results and a bibliography, see the introduction of [3].) Furthermore, a theorem of R. D. Anderson and $\mathrm{R}$. M. Schori [4] asserts that given any open cover $U$ of $M$, there is a homeomorphism $h_{\mathfrak{u}}$ of $M$ onto $M \times s$ so that $p \circ h_{\mathfrak{U}}$ is $\mathcal{U}$-close to the identity map, where $p$ is the projection onto $M$. If $\left\{s_{i}\right\}_{i=1}^{\infty}$ is a countable, indexed family of copies of $s$, it is easy to see that $s^{\prime}$, the product of the $s_{i}^{\prime}$ 's, is homeomorphic to $s$, so $s$ may be replaced by $s^{\prime}$ in the above theorem.

For each integer $i$ and real number $t$ in $(-1,1)$, let $\psi_{i, t}: s_{i} \rightarrow s_{i}$ be the map which multiplies in each coordinate by $t$, and let 


$$
\begin{aligned}
\phi(i, t) & =1 & & \text { if } t \leqq \frac{1}{i+1} \text { or } t \geqq \frac{i}{i+1}, \\
& =0 & & \text { if } \frac{1}{i} \leqq t \leqq \frac{i-1}{i}, \\
& =(i+1)(1-i t) & & \text { if } \frac{1}{i+1} \leqq t \leqq \frac{1}{i}, \\
& =(i+1)(i t-i+1) & & \text { if } \frac{i-1}{i} \leqq t \leqq \frac{i}{i+1} .
\end{aligned}
$$

Also, let $k_{i}$ be an embedding of $X$ in $s_{i}$, as a closed set if $X$ is complete. (It is well known that this may be done in a separable Banach space.)

Given any homotopy $F$ between $f$ and $g$ and any open cover $\mathcal{U}$ of $M$, let $\vartheta$ be a star-refinement of $\mathcal{U} ; h_{v}$, a homeomorphism of $M$ onto $M \times s^{\prime}$ such that $p \circ h_{v}$ and the identity are $\mathcal{V}$-close, and define $G$ : $X \times I \rightarrow M$ by

$$
\begin{aligned}
G(x, t)= & h_{v}^{-1} \circ\left[\operatorname{id}_{M} \times \prod_{i=1}^{\infty}\left(\psi_{i, \phi(i+2, t)}+\left(\psi_{i, 1-\phi(i+1, t)} \circ k_{i}(x)\right)\right)\right] \\
& \circ h_{v} \circ F(x, t),
\end{aligned}
$$

where " + " is understood to indicate coordinate-wise addition, and "II", the product of mappings.

For each $t$ in $(0,1), h_{0} \circ G \mid X \times\{t\}$ may be regarded as the product of a mapping of $X$ into $M \times \prod_{i=1, i \neq t_{0}}^{\infty} s_{i}$ with a (closed) embedding of $X$ in $s_{i_{0}}$, where $i_{0}$ is any integer greater than or equal to both $1 / t$ and $1 /(1-t)$. It is a simple matter to see that this is a (closed) embedding since it is continuous, one-to-one, and the inverse is continuous because given a point $(x, t)$ and a sequence $\left\{\left(x_{i}, t\right)\right\}_{i=1}^{\infty}$ in $X \times\{t\}$ for which $h_{0} \circ G\left(x_{i}, t\right)$ converges to $h_{0} \circ G(x, t)$, the $s_{i_{0}}$-coordinates of $\left\{h_{0} \circ G\left(x_{i}, t\right)\right\}_{i=1}^{\infty}$ converge to the $s_{i_{0}}$-coordinate of $h \circ G(x, t)$, and as the mapping into the $s_{i_{0}}$-coordinate is an embedding, this forces $\left\{x_{i}\right\}_{i=1}^{\infty}$ to converge to $x$. The image $h_{0} \circ G(X \times\{t\})$ is closed if $X$ is complete, since if $\left\{\left(x_{i}, t\right)\right\}_{i=1}^{\infty}$ is a sequence in $X \times\{t\}$ and $p$ is in $M \times s^{\prime}$ with $h_{v} \circ G\left(x_{i}, t\right)$ converging to $p$, then the $s_{i_{0}}$-coordinates of $h_{v} \circ G\left(x_{i}, t\right)$ converge to the $s_{i_{0}}$-coordinate of $p$, which forces the $s_{i_{0}}$ coordinate of $p$ to be $k_{i_{0}}(x)$, for some $x$, and thus forces $\left\{x_{i}\right\}_{i=1}^{\infty}$ to converge to $x$.

If it is desired, the pseudo-isotopy may be modified slightly to provide that 
(a) it be an embedding of $X \times(0,1)$ in $M$ and (b) the image of $X \times(0,1)$ lie in a countable union of closed sets of $M$ each of which has Property $Z$ in $M$ (in case $X$ is complete, the image of $X \times(0,1)$ may be required to be the countable union of closed sets with Property $Z$ in $M$ ).

(A closed set $Y$ has Property $Z$ in $M$ provided that for each nonnull open set $U$ of $M$ with trivial homotopy groups, $U-Y$ be also nonnull and have trivial homotopy groups. The importance of Property $Z$ for $F$-manifolds is demonstrated by [2] in which it is shown that the subsets of such which are homeomorphic to the manifolds by homeomorphisms $u$-close to the identity for all open covers $u$ are precisely the complements of countable unions of closed sets, each with Property $Z$.) The modified homotopy $H: X \times I \rightarrow M$ may be defined by setting $H(x, t)=h_{0}^{-1} \circ\left[i d_{M} \times \prod_{i=1}^{\infty} \xi_{i, t}(x)\right] \circ h_{0} \circ F(x, t)$, where

$$
\begin{aligned}
\xi_{i, t}(x) & =\psi_{i, \phi(i+2, t)}+\left(\psi_{i, 1-\phi(i+1, t)} \circ k_{i}(x)\right), \quad \text { if } i \text { is even, } \\
& =\psi_{i, \phi(i+2, t)}+\left(\psi_{i, 1-\phi(i+1, t)} \circ \psi_{i, t}\left(y_{i}\right)\right), \quad \text { if } i \text { is odd } \\
& \text { but not divisible by three, and } \\
& =\psi_{i, \phi(i+2, t)}, \quad \text { if } i \text { is an odd multiple of three. }
\end{aligned}
$$

Here, $y_{i}$ is merely a point in $s_{i}$ with not all coordinates zero; the $y_{i}$ 's are introduced for the purpose of guaranteeing that $H \mid X \times(0,1)$ be an embedding. The insertion of merely the $\psi_{i, \phi(i+2, t)}$ in infinitely many coordinates is to ensure that for any $t_{0}$ in $(0,1 / 2)$, $\left.h_{0} \circ H(X) \times\left[t_{0}, 1-t_{0}\right]\right)$ project into $s^{\prime}$ on a set of infinite co-dimension which, by a theorem of R. D. Anderson [1], must have closure with Property $Z$. This guarantees that the closure of $h_{v} \circ H\left(X \times\left[t_{0}, 1-t_{0}\right]\right)$ has Property $Z$ in $M \times s^{\prime}$ and hence that the closure of $H\left(X \times\left[t_{0}, 1-t_{0}\right]\right)$ has Property $Z$ in $M$. If $X$ is complete, the construction gives that $H\left(X \times\left[t_{0}, 1-t_{0}\right]\right)$ is closed and has Property $Z$ for each $t_{0}$ in $(0,1 / 2)$.

Remark. D. W. Henderson has recently proven in [5] that if $X$ is an $F$-manifold and $U$ an open cover of $M$, than any map of $X$ into $M$ may be approximated $U$-closely by closed and open embeddings.

In light of these results, the following question, also raised at Cornell, would seem to be the appropriate one: "Under which circumstances are two homotopic embeddings of one $F$-manifold in another ambient isotopic?"

\section{REFERENCES}

1. R. D. Anderson, On topological infinite deficiency, Michigan Math. J. 14 (1967), 365-383.

2. - Strongly negligible sets in Frechet manifolds, Bull. Amer. Math. Soc. 75 (1969), 64-67. 
3. R. D. Anderson and R. H. Bing, A complete, elementary proof that Hilbert space is homeomorphic to the countable infinite product of lines, Bull. Amer. Math. Soc. 74 (1968), 771-792.

4. R. D. Anderson and R. M. Schori, Factors of infinite-dimensional manifolds, Trans. Amer. Math. Soc. (to appear).

5. D. W. Henderson, Infinite-dimensional manifolds are open subsets of Hilbert space, Topology (to appear).

UNIVERSITY OF KENTUCKY, LEXINGTON, KENTUCKY 40506 